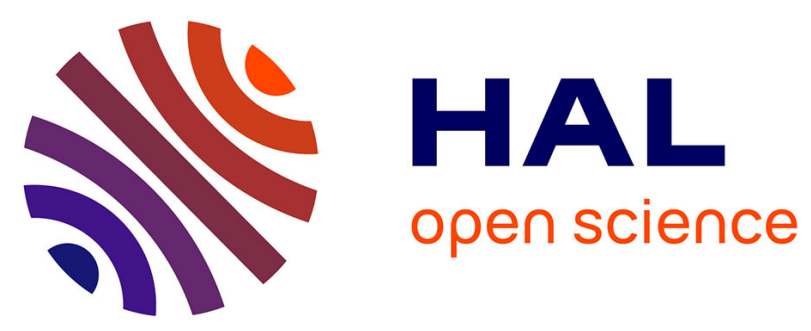

\title{
Impact on survival of early detection of isolated breast recurrences after the primary treatment for breast cancer: a meta-analysis
}

\author{
W. L. Lu, L. Jansen, W. J. Post, J. Bonnema, J. C. Velde, G. H. Bock
}

\section{- To cite this version:}

W. L. Lu, L. Jansen, W. J. Post, J. Bonnema, J. C. Velde, et al.. Impact on survival of early detection of isolated breast recurrences after the primary treatment for breast cancer: a meta-analysis. Breast Cancer Research and Treatment, 2008, 114 (3), pp.403-412. 10.1007/s10549-008-0023-4 . hal00478224

\section{HAL Id: hal-00478224 \\ https://hal.science/hal-00478224}

Submitted on 30 Apr 2010

HAL is a multi-disciplinary open access archive for the deposit and dissemination of scientific research documents, whether they are published or not. The documents may come from teaching and research institutions in France or abroad, or from public or private research centers.
L'archive ouverte pluridisciplinaire HAL, est destinée au dépôt et à la diffusion de documents scientifiques de niveau recherche, publiés ou non, émanant des établissements d'enseignement et de recherche français ou étrangers, des laboratoires publics ou privés. 


\title{
Impact on survival of early detection of isolated breast recurrences after the primary treatment for breast cancer: a meta-analysis
}

\author{
W. L. Lu · L. Jansen · W. J. Post · J. Bonnema • \\ J. C. Van de Velde · G. H. De Bock
}

Received: 29 January 2008/Accepted: 9 April 2008/Published online: 18 April 2008

(C) Springer Science+Business Media, LLC. 2008

\begin{abstract}
Purpose The purpose was to establish the impact on survival of early detection of a local recurrence of breast cancer as compared to late detection. Design A meta-analysis was carried out using Cochrane review manager software (RevMan version 4.2). Studies were included if women were treated for primary breast cancer without evidence of distant metastasis at primary diagnosis and if these concerned routine follow-up strategies focusing on the early detection of curable recurrences. Data regarding the risk for death were derived from each study. Multi level models were used to study heterogeneity by using MLWin. Results Thirteen studies concerning 2,263 patients were included. Early detection of breast cancer recurrences during follow-up gave a significantly better survival as compared to late detected recurrences (HR: 1.68 (95\% CI: 1.48-1.91)). Survival was better when the recurrence was found by mammography
\end{abstract}

W. L. Lu · W. J. Post · G. H. De Bock $(\square)$

Department of Epidemiology, University Medical Center Groningen, University of Groningen, PO Box 30001, 9700 RB Groningen, The Netherlands e-mail: G.H.de.Bock@Epi.UMCG.NL

W. L. Lu

Department of Health Statistics, School of Public Health, Tianjin Medical University, Tianjin, China

L. Jansen

Department of Surgery, University Medical Center Groningen, University of Groningen, Groningen, The Netherlands

J. Bonnema

Department of Surgery, University Hospitals Morecambe Bay, Lancaster, UK

J. C. Van de Velde

Department of Surgery, Leiden University Medical Center, Leiden, The Netherlands instead of physical examination or in patients without symptoms as compared to those with symptoms (HR: 2.44 (95\% CI: 1.78-3.35); HR: 1.56 (95\% CI: 1.36-1.79), respectively). If all breast cancer recurrences would be detected earlier, that 5-8 deaths (i.e. an absolute reduction in mortality of 17-28\%) would be avoided by performing routine follow-up during a 10 year-period for 1,000 breast cancer patients. Conclusion These data support the hypothesis that detection of isolated loco-regional or contra-lateral breast cancer recurrences in patients without symptoms has beneficial impact on survival of breast cancer patients when compared to late symptomatic detection.

Keywords Breast neoplasm - Survival · Recurrence · Early detection $\cdot$ Meta-analysis

\section{Introduction}

Screening for breast cancer has resulted in increasing numbers of patients diagnosed with early breast cancer. The combined effect of early diagnosis and improved treatment for breast cancer has led to a significant decrease in breast cancer-related mortality. As a consequence, the prevalence of breast cancer survivors rises. After curative treatment for breast cancer it is common practice to enter patients in a surveillance program for many years. There will be more breast cancer survivors followed by more need for long-term surveillance [1]. At the same time, there is an increasing pressure on breast services from new referrals and urgent cases [2]. It is predicted that there will be a $48 \%$ increased need for cancer services by 2020 . This puts an increasing burden on follow-up oncology clinics [3]. There is a need to quantify the benefits of follow-up to organise the follow-up more efficiently. 
One of the important goals of surveillance is improvement of survival. The surveillance program can extend survival when two assumptions are made: (1) most recurrences are detected at an early stage (i.e. are without symptoms at diagnosis) during the surveillance visits and (2) the early treatment of recurrences leads to better survival [4]. About $40 \%$ of loco-regional recurrences were asymptomatic at diagnosis in an earlier systemic review and meta-analysis that involved 5,045 patients and 378 isolated loco-regional recurrences [5]. The risk of breast carcinoma-related death is increased for patients with a local recurrence compared to those without local recurrence $[6,7]$. Still, the effect of early detection of curable recurrence on overall survival remains questionable and controversy remains on the benefits of regular follow-up [8]. The aim of this study is to perform a meta-analysis of the impact of early detection of loco-regional or contralateral breast cancer recurrence on survival.

\section{Methods}

\section{Search strategy}

Pubmed Medline, CancerLit, Cochrane, Web of sciences and Embase were searched for relevant studies. Studies in any language were examined published between 1966 and 2006. MESH words used were "Breast Neoplasms", "Follow-Up Studies", "Mammography", "Physical Examination" and "Survival". Title and abstract were searched for the words: "breast cancer", "follow-up", "detection", "survival" and "recurrence". Reference lists and reviews were searched by hand.

\section{Selection of papers}

Studies were included in the meta-analysis when they met the following inclusion criteria. Target population: Studies were included if women were treated for primary breast cancer without evidence of distant metastasis at primary diagnosis. Follow-up: Studies were included if they concerned routine follow-up strategies or tests focusing on the early detection of curable recurrence (loco-regional recurrence and contralateral recurrence). Contrast: Studies were included if they focused on comparing early detection (recurrences without symptoms) versus late detection (recurrences with symptoms). Outcome: Studies were included when they presented survival data. Data presentation: Studies were included when they presented hazard ratios expressing the risk of death or when they presented information for calculating these hazard ratios (number of deaths and exact p-values).
Two researchers (GHdeB and LWL) independently examined titles $(n=1,369)$ and abstracts $(n=413)$ to decide if the full text articles should be obtained. Cases of disagreement were resolved by discussing the titles and abstracts $(n=14)$. Six studies fulfilled the inclusion criteria regarding follow-up, contrast and outcome, but did not present hazard ratios or information for calculating these hazard ratios. For two of these six studies, the number of deaths among the patients with recurrences was not available [9, 10]. For two studies, no (exact) $P$-value was available [11, 12]. For two studies, the number of deaths among the patients with recurrences was not available, nor the (exact) $P$-value was available $[13,14]$. As a consequence 13 of 68 full-text articles that were examined could be included in the analysis. For an overview of studies included in the analysis, see Table 1.

\section{Data extraction and definition}

Data were extracted independently by the two researchers (GHdeB and LWL), by means of a predefined form. For an overview of the topics, see Table 1. Loco-regional recurrences were defined as the presence of cancer in the breast or axilla on the same side. Contra-lateral recurrences were defined as cancer in the other breast after the primary treatment of the first breast cancer. Distant metastases were defined as the evidence of breast cancer in any part of the body except breast and axilla. It was registered whether follow-up time was measured from the time of primary treatment or from the time of recurrence. A follow-up scheme was considered as standard when patients received regular mammography with or without physical examination. A follow-up scheme was considered as intensive when patients received additional blood tests and bone scans regularly and independent of symptoms. A recurrence was considered as being detected early when it was mammographically detected during a routine clinic visit in a patient without symptoms. A recurrence was considered as being detected late when it was detected by patient themselves.

Assessment of methodological quality and publication bias

Methodological quality was assessed independently by the investigators (GHdeB and LWL) by means of a predefined form. Because there is no generally accepted standard for measuring methodological quality in prognostic studies, this form was derived from the work of Altman and Laupacis and is presented in Table $2[15,16]$. A score six or of higher was considered as a high quality score. The cut-off point was based on the median. 


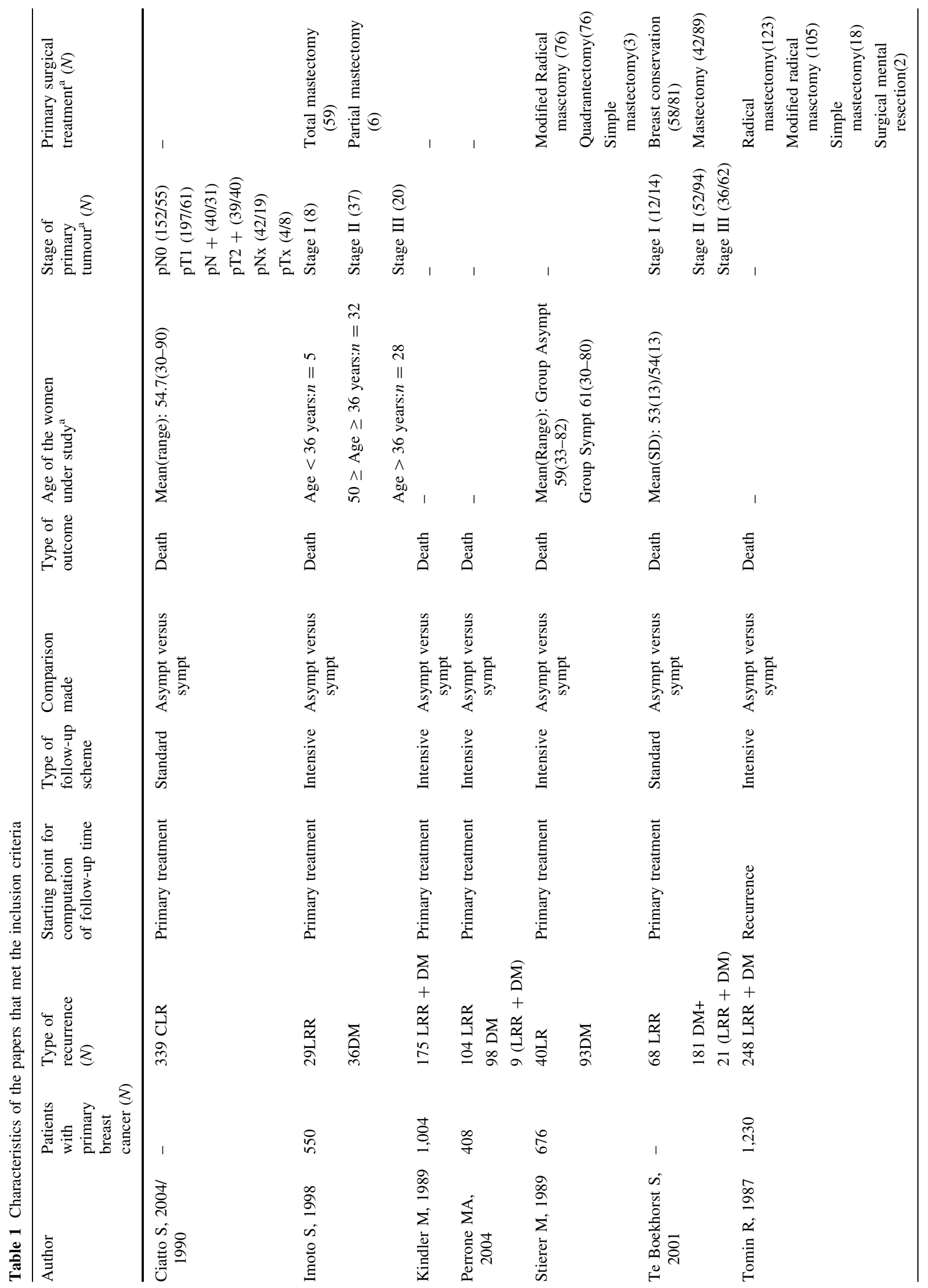




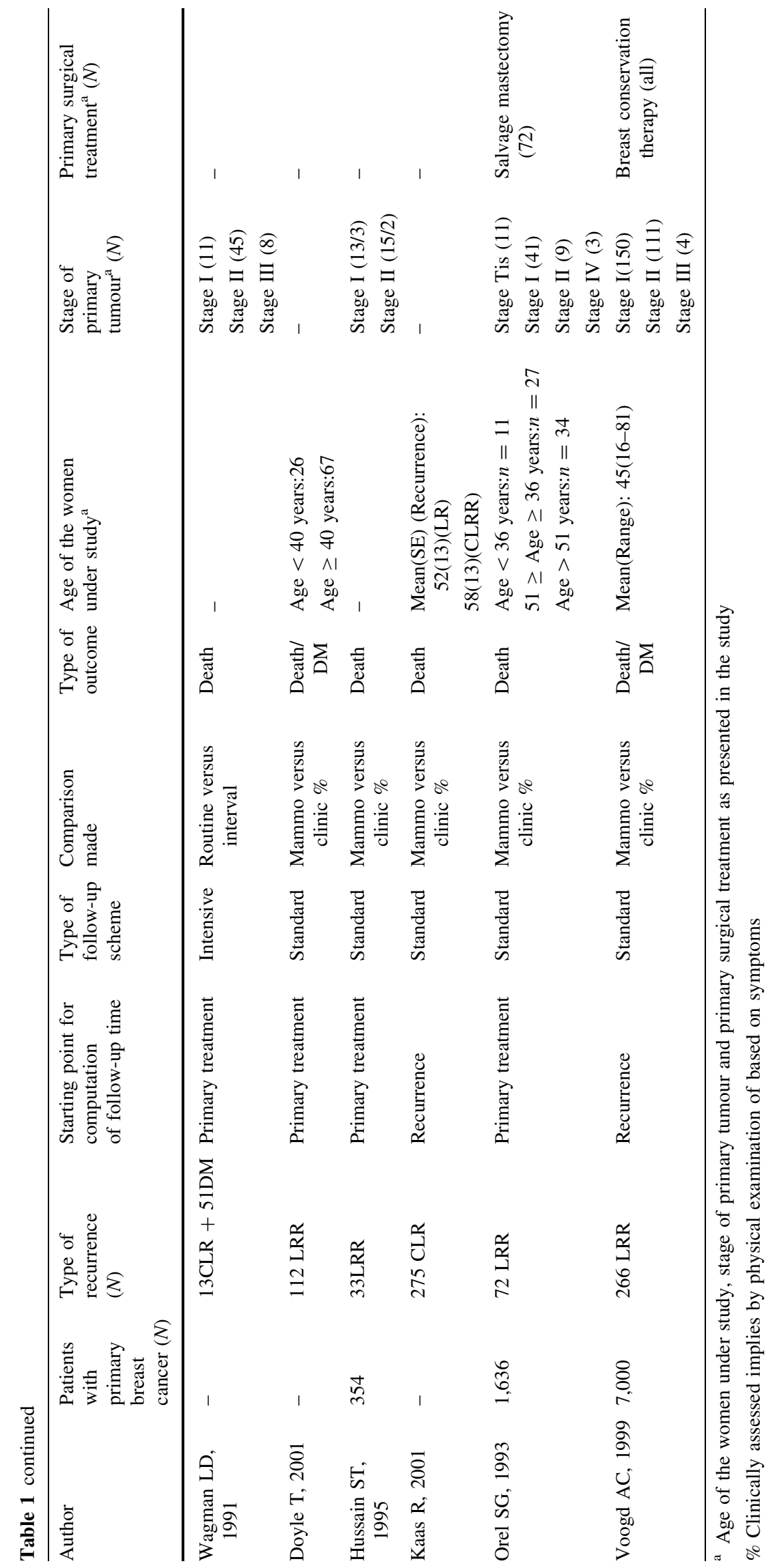


Breast Cancer Res Treat (2009) 114:403-412

407

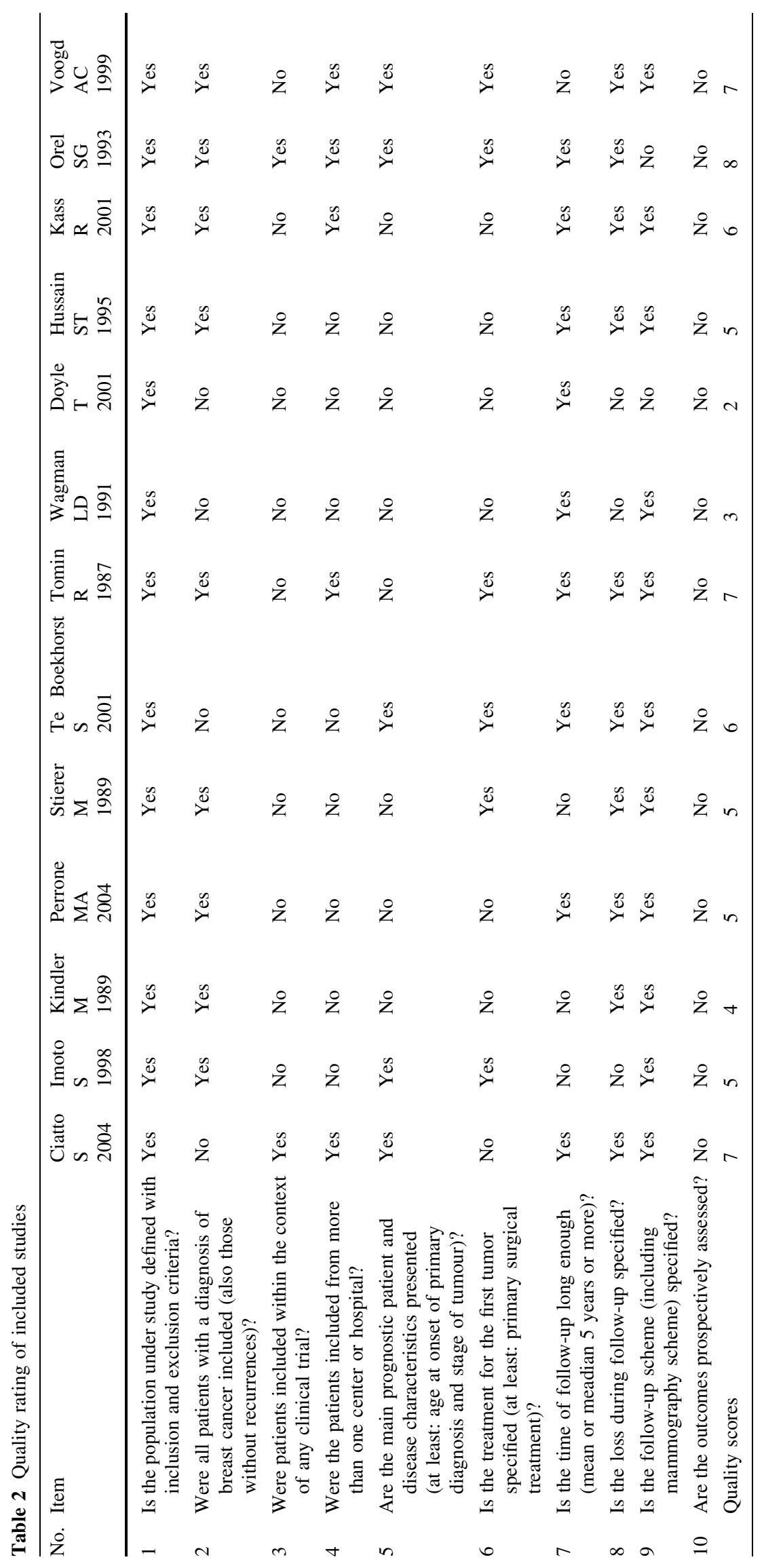

Springer 
Fig. 1 Impact on survival of early detection of recurrences after the primary treatment for breast cancer. Note: Early detected recurrences versus late detected recurrences

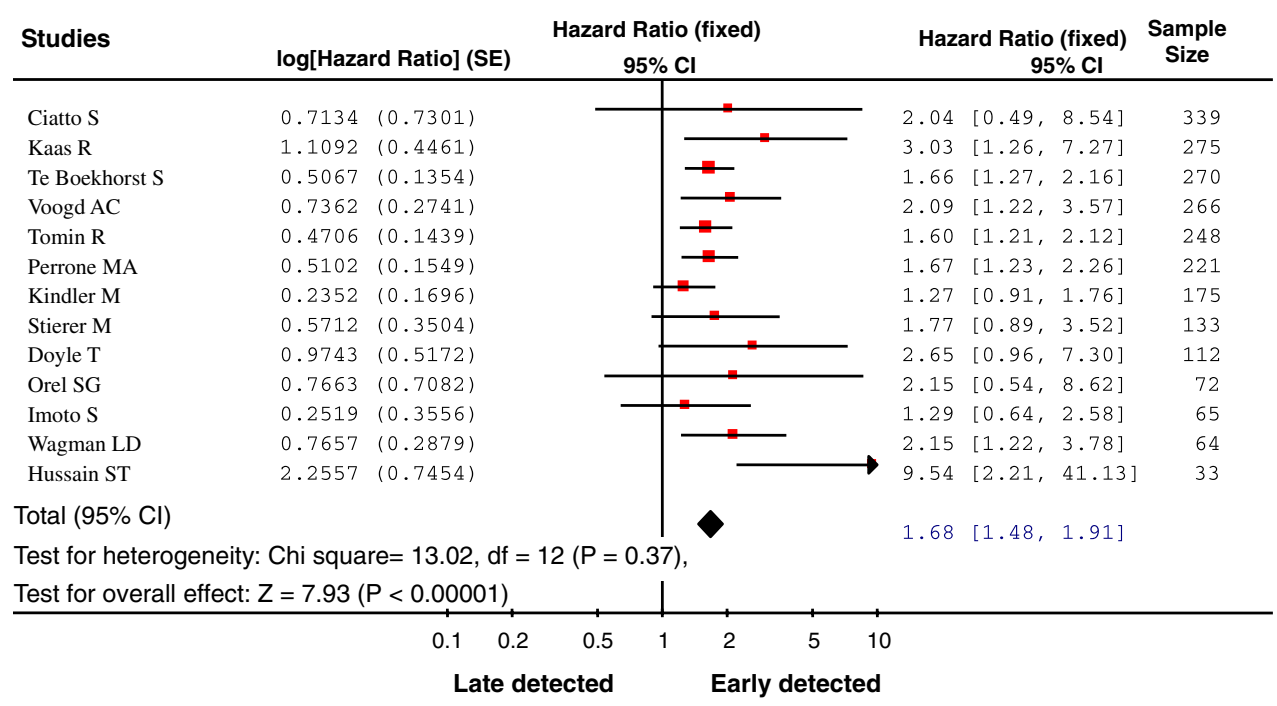

To investigate publication bias, in Fig. 1 studies are presented based on sample size to get an impression of a potential relationship between sample size and effect size. To quantify this relation, Kendall's tau coefficient was calculated [17]. To estimate whether publication bias is likely to be a problem in this meta-analysis, the fail-safe number was assessed to calculate how many studies are needed to counterbalance the results [18].

\section{Statistical analysis}

The main outcome in this analysis was the hazard ratio (HR) and its standard error (SE). If these data were not directly available, these were estimated based on the total number of events in both groups and the two-sided $P$-value by using the method described by Parmar [19]. Based on the SE, 95\% confidence intervals (CIs) were calculated. HRs were directly presented in only one study [20].

In the next step, HRs were combined by using Review Manager Version 4.2. By using the Generic Inverse Variance method, $\operatorname{logHR}$ and SErs were entered. The pooled result was expressed as combined HRs with fixed effects with a 95\% CI, and an overall test on heterogeneity was performed using the Chi-square test. Despite non significant results, we still explored heterogeneity because of the small number of studies. The following potential sources of heterogeneity were explored: the type of recurrences considered, the starting point for computation of follow-up time, type of follow-up scheme, comparisons made, quality score, and type of outcome. For each potential source of heterogeneity, a multilevel model was developed with the $\operatorname{logHR}$ as dependent variable and the source of heterogeneity as well as the SE as independent variables. To quantify the theoretical extent of survival improvement, if the loco-regional or contra-lateral recurrence would be early detected, the population attributable risk was calculated [21].

\section{Results}

Study characteristics

In the analysis, 2,263 patients from 13 studies were included having 724 loco-regional recurrences, 627 contralateral recurrences, 459 distant metastases, 30 loco-regional recurrences with distant metastases combined and 423 not specified recurrences (see Table 1). Fifty-eight percent $(1,223)$ of these patients had a recurrence detected early and $42 \%$ were with late detection. The included studies were comparable regarding the distribution of age, primary tumour stage and primary surgical treatment. In the majority of studies, the time of follow-up was measured from date of primary diagnosis $(n=10 ; 77 \%)$. In seven studies $(54 \%)$ patients were offered routine follow-up, including regular mammography and physical examination, and in six studies patients were offered intensive follow-up including routine additional tests. Seven studies (54\%) focused on patients diagnosed with recurrences without symptoms as compared to patients diagnosed with recurrences with symptoms. One study focused on recurrences found during routine follow-up or outside routine follow-up. Five studies focused on recurrences diagnosed by mammography or symptoms. Eleven studies had death as primary outcome, and two studies had a mixed outcome (death of distant metastases).

\section{Quality score and publication bias}

Seven studies had a quality score of 5 or lower (Table 2). There were no indications of publication bias because increasing sample size was not related to increasing effect size (Kendall's tau coefficient: $-0.194(P=0.36)$ ). The fail-safe number was 210 , which means 210 contrary 
Table 3 Outcomes in the papers that met the inclusion criteria

\begin{tabular}{|c|c|c|c|c|c|c|}
\hline Author & Time of follow-up & $\begin{array}{l}\text { Number of death among } \\
\text { early detected patients }\end{array}$ & $\begin{array}{l}\text { Number of death among } \\
\text { late detected patients }\end{array}$ & $\begin{array}{l}\text { Log-rank } \\
P \text {-value }\end{array}$ & HR & SE \\
\hline Ciatto S, 2004/1990 & $1-31$ years & $-/ 234$ & $-/ 105$ & 0.008 & 2.0408 & 0.7301 \\
\hline Imoto S, 1998 & Median: 878 days (196-1806) & $14.57 / 30 \triangle$ & $17.25 / 35 \triangle$ & 0.48 & 1.2865 & 0.3556 \\
\hline Kindler M, 1989 & - & $109 / 121 \triangle$ & $54 / 54 \triangle$ & 0.2 & 1.2652 & 0.1696 \\
\hline Perrone MA, 2004 & Median: 94.7 month (9.7-198.3) & $87 / 101$ & $80 / 110$ & 0.001 & 1.6656 & 0.1549 \\
\hline Stierer M, 1989 & Median:41 month & $8 / 37 \triangle$ & $26 / 56 \triangle$ & 0.1105 & 1.7705 & 0.3504 \\
\hline Te Boekhorst S, 2001 & $0-16$ years & $153 / 170$ & $81 / 100$ & 0.0003 & 1.6599 & 0.1354 \\
\hline Tomin R, 1987 & $0-16$ years & $67 / 89 \triangle$ & $143 / 159 \triangle$ & 0.0017 & 1.6010 & 0.1439 \\
\hline Wagman LD, 1991 & 5 years+ & $26 / 26 \triangle$ & $24 / 38 \triangle$ & 0.009 & 2.1505 & 0.2879 \\
\hline Doyle T, 2001 & $0-20$ years & $11 / 42$ & $4 / 47$ & 0.06 & 2.6493 & 0.5172 \\
\hline Hussain ST, 1995 & 0-11years & $14 / 28$ & $0 / 5$ & 0.03 & 9.5421 & 0.7454 \\
\hline Kaas R, 2001 & Median:82.5(10-166) & $51 / 166$ & $16 / 109$ & 0.015 & 3.0320 & 0.4461 \\
\hline Orel SG, 1993 & $0-13$ years & $6 / 38$ & $2 / 34$ & 0.28 & 2.1517 & 0.7082 \\
\hline Voogd AC, 1999 & $2-4$ years & $61 / 141$ & $10 / 47$ & 0.02 & 2.0881 & 0.2741 \\
\hline
\end{tabular}

$\triangle$ Estimated from survival curve

studies would need to be included to counterbalance the result in this meta-analysis at a significance level 0.05 .

\section{Survival}

Overall, survival was significantly better with early detection of the recurrence (HR: 1.68 (95\% CI: 1.48-1.91, $P<0.0001$; see Fig. 1). In all 13 studies, the HR showed a trend for better survival with early detection, but five out of the 13 included studies presented no significant result (Table 3). There was no significant heterogeneity among the studies (Chi-square = 13.12, $P=0.37$; Fig. 1).

The chance of benefit of early detection was statistically significant higher in the studies that presented the data regarding loco-regional recurrence and contra-lateral recurrence separately from distant metastases (HR: 2.55 (95\% CI: 1.76-3.70) as compared to the studies that did not (HR: 1.59 (95\% CI: 1.38-1.82; $P=0.02$ ); see Table 4).
Table 4 Comparison of HRs for six sources of heterogeneity

\begin{tabular}{|c|c|c|c|c|}
\hline Sources of heterogeneity & $\begin{array}{l}\text { Number of } \\
\text { studies }\end{array}$ & Combined HR & $95 \% \mathrm{CI}$ & $P$-value ${ }^{\mathrm{b}}$ \\
\hline \multicolumn{5}{|l|}{ Type of recurrence } \\
\hline Only loco-regional or contra-lateral recurrences & 6 & 2.55 & $1.76-3.70$ & \\
\hline LRR, CLR and DM & 7 & 1.59 & $1.38-1.82$ & 0.02 \\
\hline \multicolumn{5}{|l|}{ Starting point for computation of follow-up time } \\
\hline Primary treatment & 9 & 1.64 & $1.41-1.91$ & \\
\hline Recurrence & 3 & 1.77 & $1.39-2.25$ & 0.62 \\
\hline \multicolumn{5}{|l|}{ Type of follow-up scheme } \\
\hline Standard & 7 & 1.92 & $1.55-2.38$ & \\
\hline Intensive & 6 & 1.56 & $1.33-1.83$ & 0.12 \\
\hline \multicolumn{5}{|l|}{ Comparison made } \\
\hline Patient reported symptoms (no versus yes) & 7 & 1.56 & $1.36-1.79$ & \\
\hline Mammographically only versus clinically assessed ${ }^{a}$ & 6 & 2.44 & $1.78-3.35$ & 0.01 \\
\hline \multicolumn{5}{|l|}{ Quality scores } \\
\hline$>5$ & 6 & 1.73 & $1.45-2.06$ & \\
\hline$\leq 5$ & 7 & 1.81 & $1.45-2.27$ & 0.61 \\
\hline \multicolumn{5}{|l|}{ Type of outcome } \\
\hline Death or distant metastases & 2 & 2.20 & $1.37-3.54$ & \\
\hline Death & 11 & 1.64 & $1.44-1.88$ & 0.24 \\
\hline
\end{tabular}


When analyzing the studies that calculated follow-up time from the date of primary treatment separately from the studies that calculated follow-up time from the date of recurrences, we observed that the HR for the studies excluding lead-time bias were comparable for the studies that calculated time to follow-up from the date of recurrence. (HR: 1.64 (95\% CI: 1.41-1.91), HR: 1.77 (95\% CI: 1.39-2.25), respectively). Studies focusing on the impact of an intensive regimen for follow-up gave a HR for survival comparable to studies focusing on a standard regimen for follow-up (HR: 1.56 (95\% CI: 1.33-1.83), HR: 1.92 (95\% CI: $1.55-2.38)$, respectively; $P=0.12$ ). Recurrences assessed in patients without symptoms were related to a higher probability of survival than when symptoms were present (HR: 1.56 (95\% CI: 1.36-1.79)). Survival was better in studies where recurrences were found by mammography instead of studies where recurrences were being assessed clinically (HR: 2.44 (95\% CI: 1.78-3.35)). This advantage is significantly higher for studies mammographically assessed than for tumours clinically assessed $(P=0.01)$. Studies with a higher quality score were not related to a different $\mathrm{HR}$ than studies with a lower quality score (HR: 1.73 (95\% CI: 1.45-2.06), HR: 1.81 (95\% CI: 1.45-2.27), respectively; $P=0.61$ ). Studies focusing on death or distant metastases had a non-significant higher HR for survival than studies focussing on death only (HR: 2.20 (95\% CI: 1.37-3.54), HR: 1.64 (95\% CI: 1.44-1.88), respectively; $P=0.24)$.

\section{Absolute effects}

There would be an absolute reduction in mortality of $17-28 \%$ of breast cancer patients with recurrences, if all recurrences would be early detected, given the pooled HR of 1.68 (95\% CI: 1.48 to 1.91 ) and the proportion of the early detected recurrences (40\%) [5]. Given the fact that nowadays nearly all patients in Western countries are in follow-up, the incidence of recurrence (10\%) during a 10 year-period and the survival rate at 10 years $(70 \%)$ of breast cancer patients with recurrence [22], 5-8 deaths would be avoided by performing routine follow-up during a 10 year-period for 1,000 breast cancer patients.

\section{Discussion}

This meta-analysis shows that early detection of isolated recurrences in patients without symptoms by routine follow-up or mammography improves survival of patients with breast cancer recurrences $(\mathrm{HR}=1.68 ; 95 \% \mathrm{CI}$ : 1.48-1.91). Given the proportion of early detected locoregional recurrences is $40 \%$, there would be an absolute reduction in mortality of $17-28 \%$ of breast cancer patients with recurrences, if all loco-regional recurrences would be detected early. Individual studies have been inconclusive in answering the question whether early detection of breast cancer recurrences is related to longer life. Five studies included did not show a significant difference in improving survival between early detection versus late detection of loco-regional recurrence [23-27]. One explanation might be that these studies had too small sample sizes. The metaanalysis allowed us to include the observations of more than 2,000 patients, and yields a far more precise estimate of the effect on survival of early detection of loco-regional or contra-lateral breast cancer recurrences.

A topic of debate in follow-up of breast cancer is the role of mammography and physical examination. Several studies concluded that the early detection of local disease recurrence require both clinical examination and mammography [28-30]. One study reported that the tumour size of local recurrences detected by mammography alone were smaller than those detected by physical examination [29]. Several studies [20, 31, 32] suggested that breast cancer patients who received regular mammograms were less likely to die than breast cancer patients who did not, and that recurrences found by mammography are associated with a better survival $[33,34]$. A similar effect was seen in this meta-analysis. Our findings suggested that survival is better when the recurrence is found by mammography instead of physical examination (HR: 2.44 (95\% CI: $1.78-$ $3.35)$ ). There were insufficient data to study the contribution of yearly mammogram as compared to 6 month mammogram. In this meta-analysis, late detected recurrences included those recurrences detected by breast selfexamination or by symptoms. Recurrences assessed in patients without symptoms are also related to a higher probability of survival than when symptoms are present (HR: 1.56 (95\% CI: 1.36-1.79)). This advantage is higher for tumours assessed by mammography $(P=0.01)$. These findings indicate that the actual survival benefit of early detection of a local recurrence by mammography may be higher than the pooled overall data reported in this metaanalysis. In the absence of related studies, the contribution of breast self-examination is not clear. According to the favorable effect size of early detection of curable recurrence by mammography, the result is in line with ASCO guideline that women should be made aware that monthly BSE does not replace mammography as a breast cancer screening tool to early detect isolated breast recurrences after a primary treatment for breast cancer [35].

In this meta-analysis, some studies were included that did not present data for loco-regional recurrence and contralateral recurrence separately from distant metastases, although the primary focus was loco-regional recurrence or contralateral recurrence [8, 24-26, 36, 37]. We found that the chance of benefit of early detection is significantly 
higher in the studies that presented the data regarding locoregional recurrence and contralateral recurrence separately from distant metastases (HR: 2.55 (95\% CI: 1.76-3.70) as compared to the studies that did not (HR: 1.59 (95\% CI: $1.38-1.82 ; P=0.02)$ ). The combined HR was attenuated in the studies which included distant metastasis because the early detection of distant metastasis unlikely had benefit on survival $[38,39]$.These findings may indicate that the actual survival benefit of early detection of a local recurrence is even higher than found in this meta-analysis.

There was no statistically significant difference in HR for survival between studies in this meta-anlaysis that used an intensive regimen compared to a standard regimen for follow-up (HR: 1.92 (95\% CI: 1.55-2.38; HR: 1.56 (95\% CI: $1.33-1.83)$, respectively). This is in line with previous publications in which it is found that intensive follow-up schemes focusing on the early detection of distant metastases does not improve the chances of survival $[38,39]$.

Lead time bias may have influenced the outcome of an analysis like we did [37, 40]. Lead time bias means that patients with disease detected by early diagnosis survive longer than those whose disease is detected on the occurrence of new signs or symptoms, even when treatment is without effect. When analyzing the ten studies that calculated follow-up time from the date of primary treatment separately from the three studies that calculated follow-up time from the date of recurrences we observed that the HR for the studies excluding lead-time bias is comparable with the HR for the studies that calculated time to follow-up from the date of recurrence. (HR: 1.64 (95\% CI: 1.411.91), HR: 1.77 (95\% CI: 1.39-2.25, respectively). Studies focusing on death or distant metastases had a non-significant higher HR for survival than studies focussing on death only (HR: 2.20 (95\% CI: 1.37-3.54), HR: 1.64 (95\% CI: $1.44-1.88$ ), respectively; $P=0.24$ ). This means that in this analysis, the effect of lead-time bias does not explain the effect of early detection that we found.

Studies with a higher quality score were not related to better outcome than studies with a lower quality score (HR: 1.73 (95\% CI: 1.45-2.06), HR: 1.81 (95\% CI: 1.45-2.27), respectively; $P=0.24$ ). Identical benefits were presented in studies with high quality scores and studies with low quality scores. Increasing sample size was not related to increasing effects size (Kendall's tau coefficient: $-0.194(P=0.36)$ ). The fail-safe number of 210 indicates that 210 contrary studies would be needed to counterbalance the result in this metaanalysis at a significance level 0.05 . So even if publication bias existed, it is not a problem that weakened the results of this meta-analysis.

A limitation of this meta-analysis is that all included studies were retrospective. The optimal design would be a clinical trial in which patients are randomized to follow-up versus no-follow-up. Such a study is not feasible for ethical and psychological reasons. Besides the early detection of recurrence, there are many other factors that have impact on breast cancer patients' survival. Due to the incomplete information on some important prognostic factors like age of the women, tumour stages or surgical treatment, we were not able to analyze the impact of these factors on the survival related to early detection of recurrences.

These data support the hypothesis that detection of breast cancer loco-regional or contra-lateral recurrences in asymptomatic patients during routine follow-up or assessed by mammography improves survival, when compared to late symptomatic detection and give an indication of the absolute effect. Further studies should focus on improvement of follow-up strategies aiming at early detection of loco-regional or contra-lateral recurrences and on costeffectiveness of these strategies.

\section{References}

1. Parkin DM, Fernandez LM (2006) Use of statistics to assess the global burden of breast cancer. Breast J 12(Suppl 1):S70-S80

2. Hiramanek N (2004) Breast cancer recurrence: follow up after treatment for primary breast cancer. Postgrad Med J 80:172-176

3. Erikson C, Salsberg E, Forte G et al (2007) Future supply and demand for oncologists-challenges to assuring access to oncology services. J Oncol Practice 3:79-86

4. Schapira DV, Urban N (1991) A minimalist policy for breast cancer surveillance. JAMA 265:380-382

5. De Bock GH, Bonnema J, Van Der Hage J et al (2004) Effectiveness of routine visits and routine tests in detecting isolated locoregional recurrences after treatment for early-stage invasive breast cancer: a meta-analysis and systematic review. J Clin Oncol 22:4010-4018

6. Kemperman H, Borger J, Hart A et al (1995) Prognostic factors for survival after breast conserving therapy for stage I and II breast cancer. The role of local recurrence. Eur J Cancer 31:690-698

7. Clarke M, Collins R, Darby S et al (2005) Early Breast Cancer Trialists' Collaborative Group (EBCTCG). Effects of radiotherapy and of differences in the extent of surgery for early breast cancer on local recurrence and 15-year survival: an overview of the randomised trials. Lancet 366:2087-2106

8. Te Boekhorst DS, Peer NG, van der Sluis RF et al (2001) Periodic follow-up after breast cancer and the effect on survival. Eur J Surg 167:490-496

9. Rutgers EJ, van Slooten EA, Kluck HM (1989) Follow-up after treatment of primary breast cancer. Br J Surg 76:187-190

10. Dewar JA, Kerr GR (1985) Value of routine follow up of women treated for early carcinoma of the breast. Brit Med J (Clin Res Ed) 291:1464-1467

11. Ciatto S, Rosselli Del Turco M, Pacini P et al (1985) Early detection of breast cancer recurrences through periodic followup-is it useless? Tumori 71:325-329

12. Broyn T, Froyen $\mathbf{J}$ (1982) Evaluation of routine follow-up after surgery for breast carcinoma. Acta Chir Scand 148:401-404

13. Krengli M, Pastore G, Maffei S (1993) The importance of the follow-up in patients operated on for breast cancer. A retrospective analysis of 2,482 cases. Minerva Med 84:409-415

14. Pivot X, Asmar L, Hortobagyi GN et al (2000) A retrospective study of first indicators of breast cancer recurrence. Oncology 58:185-190 
15. Altman DG, Lyman GH (1998) Methodological challenges in the evaluation of prognostic factors in breast cancer. Breast Cancer Res Treat 52:289-303

16. Laupacis A, Wells G, Richardson WS et al (1994) Users' guides to the medical literature, $\mathrm{V}$ : how to use an article on prognosis. JAMA 272:234-237

17. Begg CB, Mazumdar M (1994) Operating characteristics of a rank correlation test for publication bias. Biometrics 50: 1088-1101

18. Rosenberg MS (2005) The file drawer problem revisited: a general weighted method for calculating fail-safe number in metaanalysis. Evolution Int J Org Evolution 59:464-468

19. Parmar MKB, Torri V, Stewart L (1998) Extracting summary statistics to perform meta-analyses of the published literature for survival endpoint. Statist Med 17:2815-2834

20. Ciatto S, Miccinesi G, Zappa M (2004) Prognostic impact of the early detection of metachronous contralateral breast cancer. Eur J Cancer 40:1496-1501

21. Breslow NE, Day NE (1980) Statistical methods in cancer research. Volume I-The analysis of case-control studies. IARC Sci Publ 32:5-338

22. Soerjomataram I, Louwman MW, Ribot JG, Roukema JA, Coebergh JW (2008) An overview of prognostic factors for longterm survivors of breast cancer. Breast Cancer Res Treat 107: 309-330

23. Doyle T, Schultz DJ, Peters C et al (2001) Long-term results of local recurrence after breast conservation treatment for invasive breast cancer. Int J Radiat Oncol Biol Phys 51:74-80

24. Imoto S, Jitsuiki Y (1998) Detection of the first recurrence during intensive follow-up of breast cancer patients. Jpn J Clin Oncol 28:597-600

25. Kindler M, Steinhoff G (1989) Follow-up of breast cancer patients. Oncology 46:360-365

26. Stierer M, Rosen HR (1989) Influence of early diagnosis on prognosis of recurrent breast cancer. Cancer 64:1128-1131

27. Orel SG, Fowble BL, Solin LJ et al (1993) Breast cancer recurrence after lumpectomy and radiation therapy for early-stage disease: prognostic significance of detection method. Radiology 188:189-194

28. Wagman LD, Sanders RD, Terz JJ et al (1991) The value of symptom directed evaluation in the surveillance for recurrence of carcinoma of the breast. Surg Gynecol Obst 172:191-196
29. Voogd AC, van Tienhoven G, Peterse HL et al (1999) Local recurrence after breast conservation therapy for early stage breast carcinoma: detection, treatment, and outcome in 266 patients. Dutch Study Group on Local Recurrence after Breast Conservation (BORST). Cancer 85:437-446

30. Mellink W, Holland R, Hendrisks T (1991) The contribution of routine follow-up mammography to an early detection of a synchronous contralateral breast cancer. Cancer 67:1844-1848

31. Lash TL, Clough-Gorr K, Silliman RA (2006) Reduced mortality rate associated with annual mammograms after breast cancer therapy. Breast J 12:2-6

32. Kaas R, Hart AA, Besnard AP et al (2001) Impact of mammographic interval on stage and survival after the diagnosis of contralateral breast cancer. Br J Surg 88:123-127

33. Montgomery DA, Krupa K, Jack WJL et al (2007) Changing pattern of the detection of locoregional relapse in breast cancer: the Edinburgh experience. Brit J Cancer 96:1802-1807

34. Hussian ST, Gui GP, Lee KS et al (1995) Detection of locoregional recurrence after breast-conserving surgery and radiotherapy. J R Coll Surg Edinb 40:163-166

35. Khatcheressian JL, Wolff AC, Smith TJ et al (2006) American Society of Clinical Oncology 2006 update of the breast cancer follow-up and management guidelines in the adjuvant setting. J Clin Oncol 24:5091-5097

36. Perrone MA, Musolino A, Michiara M et al (2004) Early detection of recurrences in the follow-up of primary breast cancer in an asymptomatic or symptomatic phase. Tumori 90:276-279

37. Tomin R, Donegan WL (1987) Screening for recurrent breast cancer-its effectiveness and prognostic value. J Clin Oncol 5: $62-67$

38. Rosselli Del Turco M, Palli D, Cariddi A et al (1994) Intensive diagnostic follow-up after treatment of primary breast cancer. A randomized trial. National Research Council Project on Breast Cancer follow-up. JAMA 271:1593-1597

39. The GIVIO Investigators (1994) Impact of follow-up testing on survival and health-related quality of life in breast cancer patients. A multicenter randomized controlled trial. JAMA 271: $1587-1592$

40. Ciatto S, Ambrogetti D, Bonardi R et al (1990) Prognostic impact of early detection of contralateral primary breast cancer. Tumori 76:370-373 